\title{
Innovative Technologies in Experimental Comeal Surgery ex vivo
}

\author{
DOI: $10.17691 / \mathrm{stm} 2018 \cdot 10.4 .10$
}

Received July 3, 2018

O. Yu. Komarova, PhD Student, Department of Transplantation and Optical Reconstructive Surgery

of Anterior Segment of the Eyebalt!

K.E. Lapshin, Researcher2;

K.V. Burdel, Ohthalmologist:

A.V. Shatskikh, MD, PhD, Head of the Laboratory of Eye Pathology and Histology ${ }^{1}$; Pathologist ${ }^{1}$;

M.V. Zimina, PhD Student, Department of Transplantation and Optical Reconstructive Surgery

of Anterior Segment of the Eyeball ${ }^{1}$;

A.N. Pashtaev, MD, PhD, Researcher, Department of Transplantation and Optical Reconstructive Surgery

of Anterior Segment of the Eyeball;

S.V. Novikov, Deputy Director-General in Charge of Production ${ }^{3}$;

S.K. Vartapetov, Leading Researcher2;

S.B. Izmailova, MD, DSc, Head of the Department of Transplantation and Optical Reconstructive Surgery of Anterior Segment of the Eyeball ${ }^{1}$

1S. Fyodorov Eye Microsurgery Federal State Institution, 59a Beskudnikovsky Blvd, Moscow, 127486, Russia;

${ }^{2}$ Center for Physical Instrumentation, Prokhorov General Physics Institute of the Russian Academy of Sciences,

Moscow, Troitsk, 108840, Russia;

${ }^{3}$ Scientific-Experimental Production "Eye Microsurgery" Ltd., 59a Beskudnikovsky Blvd, Moscow, 127486, Russia

The aim of the study was to assess the feasibility and outcomes of surgical interventions on the cornea in ex vivo experiments using the Femto Visum femtosecond laser system (Optosystems Ltd., Russia).

Materials and Methods. This collective study was conducted by the S. Fyodorov Eye Microsurgery Federal State Institution together with the Scientific-Experimental Production "Eye Microsurgery" Ltd. We have developed a technology for surgical intervention on the cornea with the polymeric intrastromal ring implantation aimed to prevent post-keratoplastic astigmatism. To correct defects of the iris, an original colored gel implant was inserted into the corneal tunnel. With the participation of Optosystems Ltd., we developed an algorithm and related software for using femtolaser keratotomy in the treatment of progressive keratoconus. These interventions were performed with 23 donor (cadaver) eyes that had been earlier rejected for a possible use in human keratoplasty. To assess the feasibility of the posterior lamellar keratoplasty using Femto Visum, we tested the surface quality of the ultrathin grafts prepared from 10 isolated corneal flaps.

Results. The proposed keratoplasty technology and the implantation of the intrastromal ring resulted in a complete corneal penetration free from adverse tissue bridges or adhesions. Following the kerato-pigmentation procedure, the gel-colored implant was found stably held in the preformed corneal tunnel; the remaining cornea areas remained intact. The femtolaser-assisted keratotomy resulted in radial incisions at the pre-determined locations. According to the roughness parameter, the surface quality of the ultrathin grafts produced with the femtosecond laser assistance was similar to that of the standard samples produced with a mechanical microkeratome.

Conclusion. The use of the Femto Visum femtosecond laser system in innovative areas of corneal surgery is effective and safe.

Key words: keratoplasty; intrastromal ring; kerato-pigmentation; keratoconus; femtolaser keratotomy; ultrathin graft; femtosecond laser.

\section{Introduction}

This article presents the results of ex vivo research into the use of a Femto Visum femtosecond laser (Optosystems Ltd., Russia) in corneal surgery. The studies covered four areas: 1) prevention of postkeratoplastic astigmatism; 2) kerato-pigmentation for the correction of iris defects; 3 ) femtolaser keratotomy in the treatment of progressive keratoconus; 4) qualities of femtolaser-produced ultrathin graft for posterior lamellar keratoplasty.
1. With the transparent engraftment following penetrating keratoplasty (PK) or deep anterior lamellar keratoplasty (DALK), postoperative astigmatism is a major cause of the decrease in patient's vision. Among the astigmatism prevention measures are: a) trephination systems and a femtosecond laser that provide for a more even and high-quality trephination of the recipient cornea and the donor corneoscleral flap, b) changing the cut profile according to different patterns ("mushroom", "zigzag"), and c) changing the technique of

Corresponding author: Olga Yu. Komarova, e-mail: ol.komarova91@gmail.com 
transplant suturing. Earlier, a method for carrying out the PK/DALK manipulations was proposed; there, a corneal intrastromal ring (made of a titanium alloy with other metals) was placed into the interface between the donor cornea and the cornea of the recipient [1]. In further studies, no significant difference in post-keratoplastic astigmatism was found between patients implanted and non-implanted with the intrastromal ring [2]. Another method to prevent postoperative astigmatism was described: that included simultaneous implantation of intrastromal segments into the donor cornea disk within the PK or DALK interventions [3]. Despite using these preventive measures, the incidence of astigmatism in the postoperative period continues to be high, which necessitates a search into new means of prevention.

2. There are various methods for correcting iris defects: among them, extraocular - colored contact lenses, corneal - kerato-pigmentation, intrastromal implants, intraocular — artificial iris, intraocular lenses with colored haptics. Intra-corneal implants were first proposed by Choyce; they were blue-colored perforated polymethyl methacrylate ring-shaped plates [4]. The implants were placed in a corneal pocket preformed manually. The method has several disadvantages: low diaphragm adjustability, mutual incompatibility between the implant and cornea profiles, and suture protrusions [5]. During the formation of a corneal pocket and the insertion of an implant, the central optical zone can also be affected, which increases the risk of developing secondary corneal dystrophy.

In 1986, a colored hydrogel for intra-corneal implantation was developed in the S. Fyodorov Eye Microsurgery Federal State Institution. All manipulations were performed in the cornea and did not affect the intraocular space. This technology had some drawbacks: e.g., poor permeability and low diaphragm adjustability [6]. Kerato-pigmentation (tattooing of the cornea) as a cosmetic procedure has been performed for a long time; yet the intraoperative use of mineral pigments for therapeutic purposes has been reported just recently. Superficial and intrastromal (manual or femtolaserassisted) kerato-pigmentation techniques have been described in the literature [7]. Most of patients with iris defects are socially active people of working age; therefore, their rehabilitation is a matter of priority. In these people, glare-associated poor vision reduces the quality of life. Artificial iris implantation is not the treatment of choice in many patients because of its inevitable traumatic complications. Of interest is the possibility of correcting iris defects after iridectomy for neoplasm, since the implantation of an artificial iris is undesirable in these patients because of postoperative injury. The corneal method of correcting aniridia by inserting a gel-colored implant into the area above the iris defect makes it possible to avoid intraocular intervention.

3. The method of radial keratotomy for the correction of myopia and myopic astigmatism became common in the second half of the $20^{\text {th }}$ century. Currently, this type of intervention has been superseded by more effective methods using laser correction; however, in the current ophthalmic surgery, this treatment is still used to correct ametropia in patients with keratoconus. The efficacy of this treatment was confirmed by the results of international [8, 9] and Russian studies [10, 11]. In 1997, the Italian ophthalmologist Lombardi and his associates reported the efficacy of asymmetric radial keratotomy in patients with keratoconus [12]. Later, this approach was further developed so that in 2016 Abbondanza presented the results of a retrospective study on asymmetric radial keratotomy followed by UV-crosslinking; that study indicated the possibility of correcting the refraction abnormalities and stabilizing the keratectatic process [13]. There are additional reports on the efficacy of radial keratotomy in patients with keratoconus [14-16]. However, this approach is not free from complications, such as incomplete scarring in the area of incisions and an adverse epithelial growth into the area of incisions with the formation of epithelial plugs and subepithelial fibrosis; the above complications may compromise the treatment outcome and cause a subsequent decrease in visual ability [15-17]. In mechanical radial keratotomy, the formation of micro- and macro-perforations is also possible [18]. The cited studies indicate the efficacy of radial keratotomy in patients with keratoconus, and also warn against possible complications that may occur with this type of technology. Taken together, the above results stimulate further research and development of improved corneal surgery techniques, including those based on a femtosecond laser.

4. Endothelial epithelial dystrophy (EED) of the cornea of various etiologies (secondary EED, Fuchs dystrophy, iridocorneal endothelial syndrome) is commonly accepted as indications for keratoplasty. Automated posterior keratoplasty, also known as DSAEK (Descemet's stripping automated endothelial keratoplasty), is practically the gold standard for the treatment of EED; however, the best results in visual rehabilitation can be obtained only with the socalled ultrathin graft, which has a central thickness not exceeding $130 \mu \mathrm{m}$. Producing such a transplant by the standard method - i.e., by a mechanical microkeratome - is associated with technical difficulties resulting in a thicker corneal graft (which affects the visual functions) or in a perforation, after which the product is rejected [19-21]. An alternative is using a femtosecond laser. In general, this technique is more accurate, but when forming a graft from the front surface of the cornea, it has the same drawbacks as the traditional microkeratome technique. Among those, a thick peripheral part as compared with the central part that may cause a hypermetropic refraction shift and a dislocation in the postoperative period. Another problem is the poor predictability of the final graft thickness due to the fact that the donor material stored in a conservation medium absorbs water, which affects the accuracy of 
the ultrasound and optical methods used to measure the thickness of the donor cornea. In addition, some inaccuracy can arise due to the laser radiation tuning to greater depths [22, 23]. To solve the above issues, producing a graft from the endothelial side of the cornea has been discussed.

The aim of the study was to assess the feasibility and outcomes of surgical interventions on the cornea using a Femto Visum laser in ex vivo experiments.

\section{Materials and Methods}

The proposed method for the prevention of postoperative astigmatism includes the implantation of the original intrastromal ring (ISR) into the bed of the recipient's cornea pretreated with a femtosecond laser. Together with the Scientific-Experimental Production "Eye Microsurgery" Ltd. science and production unit, we developed an ISR polymeric prototype with an external diameter of 6.5 to $8.5 \mathrm{~mm}$. We also proposed a technique for grading the trephination incisions. Simultaneously with performing PK/DALK (of the "mushroom" profile) on the residual peripheral part of the cornea, we created an intralamellar pocket. The ISR was implanted into this pocket while making sure that the external ISR diameter fitted the external diameter of the pocket. Then, the graft was fixed to the corneal bed with a 10/0 nylon blanket suture. The ISR stayed attached to the surrounding tissues in the intralamellar pocket, which eliminated the need for its suturing $[24,25]$. The surgery was performed ex vivo using isolated donor (cadaver) eyes. Total of 8 eyes were used to develop and practice the technique: in four of them, PK manipulations with ISR implantations were performed, and in the other four - the DALK procedures with ISR implantations. Depending on the thickness of the cornea (measured with OCT prior to the operation), the depth of the "step" in the "mushroom" pattern was determined - it equaled $2 / 3$ of the cornea thickness in a given zone.

The kerato-pigmentation procedure was performed on 7 donor eyes not suitable for human keratoplasty as determined by experts in the tissue bank of the S. Fyodorov Eye Microsurgery Federal State Institution. Using a femtosecond laser (in the "Corneal tunnel" mode), a ring-shaped closed corneal tunnel with an external diameter of $8.5 \mathrm{~mm}$ and an internal diameter of $5.0 \mathrm{~mm}$ was created at a depth of $400 \mu \mathrm{m}$ [26]. The incision made for the tunnel entry had a length of $3.5 \mathrm{~mm}$. The cornea thickness of the cadaver eyes was $850-900 \mu \mathrm{m}$. An aliquot of $0.5 \mathrm{ml}$ of the gel implant was placed into the corneal tunnel using a cannula.

For laser radial keratotomy, specialists from S. Fyodorov Eye Microsurgery Federal State Institution and Optosystems Ltd. developed an algorithm to control the movement of the femtosecond laser focal spot as well as the supportive software for implementing this type of operation using the Femto Visum instrument. The implementation of such an algorithm is associated with technical complexity due to the simultaneous movement of a laser beam in three dimensions with a high speed and positioning accuracy. It is worth mentioning that the laser is capable of interacting with the largest operating area - with a diameter of more than $12 \mathrm{~mm}$. Thus, the outer diameter of the corneal incisions is limited only by the limbus, provided that the required applanation is reached.

In our experimentation on 8 cadaver eyes (found unsuitable for human keratoplasty in S. Fyodorov Eye Microsurgery Federal State Institution), the femtolaser keratotomy procedure using a Femto Visum laser was developed and patented [27]. To this end, 8 radial incisions were made on the 8 cadaver eyes in the annular zone with an outer diameter of $9.5 \mathrm{~mm}$ and an inner diameter of $4.0-5.0 \mathrm{~mm}$. The depth of the incisions was $85 \%$ of the minimum kerato-pachymetry value according to OCT. The distance from the anterior surface of the cornea was $90-200 \mu \mathrm{m}$ without penetrating the Bowman's membrane and $45 \mu \mathrm{m}$ with the penetration. The thickness of the epithelial layer was determined using OCT.

Before and after carrying out the above manipulations, OCT of the cornea was performed (Visante OCT instrument, Carl Zeiss, Germany) together with photo registration (a photo slit lamp, Haag-Streit, Germany).

The experimental material was fixed in a solution of neutral formalin, washed with running water, dehydrated in alcohol of increasing concentration, and then embedded in paraffin. Histological sections stained with hematoxylin and eosin were made and the obtained preparations were examined under a Leica DM microscope (Leica Microsystems, Germany) at x50, $x 100, x 200$, and x400 magnification followed by photo registration.

The surface quality of the ultrathin grafts for posterior lamellar keratoplasty was examined in 10 corneal samples. The control group included 5 corneal flaps obtained in the course of graft production using a mechanical microkeratome (Moria SLK 2; Moria, France). In the study group, 5 corneal discs were obtained in the course of graft production using a femtosecond laser from the endothelial access. The horizontal cut was performed at a depth of $130 \mu \mathrm{m}$. The stroma surface of the donor cornea congruent of the graft was scanned. The donor cornea was stored in the Borzenko-Moroz conservation medium.

When cutting out an ultrathin graft, the donor cornea samples were placed in $10 \%$ formalin. Immediately before the test, they were freeze-dried by the standard method (FreeZone; Triad Labconco, USA). The obtained dehydrated samples were examined using a Certus V atomic force microscope (Nano Scan Technology, Russia) in the contact mode in air; for microscopy we used probes (MSCT-AUNM, Veeco, USA) with a beam stiffness of $0.01 \mathrm{~N} / \mathrm{m}$ and a curvature radius of $10 \mathrm{~nm}$. A quantitative morphometric analysis was performed using the standard microscope software. Before 
calculating the root-mean-square surface roughness (RMS), the obtained images were straightened by the software along the $x$ and $y$ axes. Moreover, for every sample, at least 5 images with an area of $400 \mu \mathrm{m}^{2}$ $(20 \times 20 \mu \mathrm{m})$ each were analyzed. The statistical significance of the differences was calculated using the non-parametric Mann-Whitney test. A confidence coefficient of $p<0.05$ was considered significant.

\section{Results}

In our keratoplasty experiments with the implantation of an original ISR, the ring remained stabile at the predetermined depth (the OCT data, Figure 1).

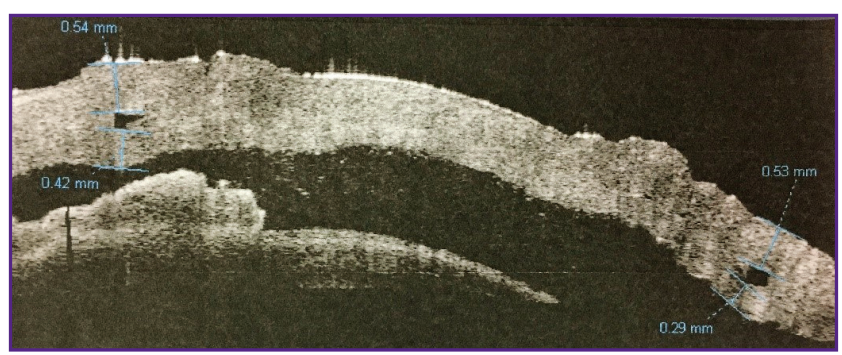

Figure 1. OCT: a sagittal section of the donor eye The DALK procedure with ISR implantation has been performed; note the uniform throughout position of the ISR
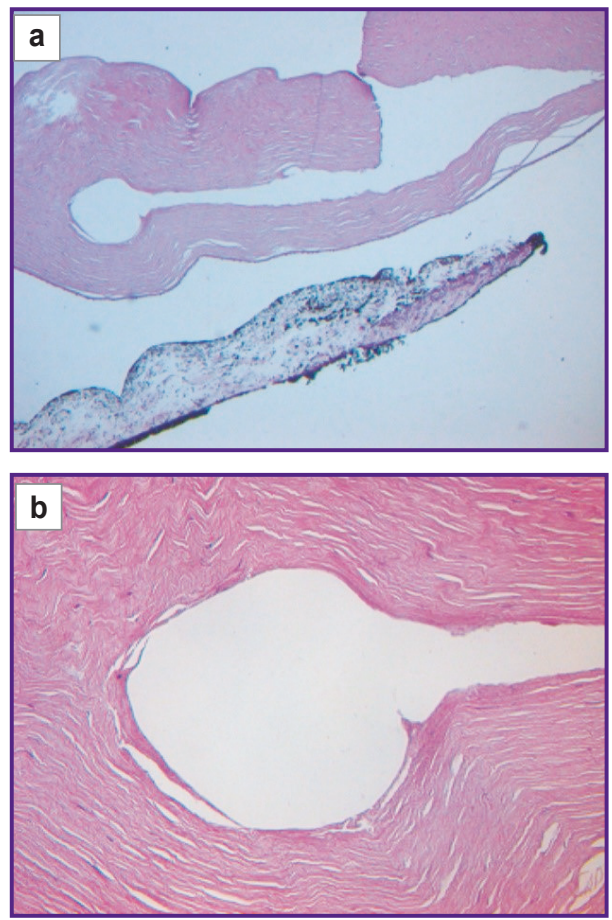

Figure 2. Histological sample of a de-epithelized donor cornea after DALK simulation involving a femtolaser Lateral intralamellar pocket; note the peripheral expansion due to the installation of the ISR; hematoxylin and eosin staining; (a) $\times 50$; (b) $\times 200$
In the histological assays, complete corneal penetration without adverse tissue bridges or adhesions was noted; the implant shape fitted precisely to that set by the femtosecond laser (Figure 2).

The visual assessment showed that the gel colored implant was successfully held in the corneal tunnel created by the femtolaser. The remaining areas of the cornea remained intact (Figure 3).

The gel implant was evenly spread throughout the corneal tunnel; when positioned this way, it had a shielding effect (Figure 4). Histological examination of the brown gel dye injected into the corneal tunnel showed peripheral pigmentation (Figure 5).

Our experimental femtolaser keratotomy resulted in 8 radial, symmetrical in length and thickness, equidistant incisions situated perpendicular to the surface of the cornea. The central and limbal zones of the cornea remained intact. There were no gas bubbles in the anterior chamber, indirectly indicating that the integrity of the Descemet membrane was preserved (Figure 6). Histological examination of the cornea samples after femtosecond laser keratotomy revealed changes in the corneal tissue typical of the femtolaser effect. Namely, the intrastromal tissue was packed tighter along the cutting edge, where the laser impact was the highest and where an intrastromal cavity formed by the gas bubbles evolved. The stroma looked dissected to a predetermined depth throughout, the indentations from

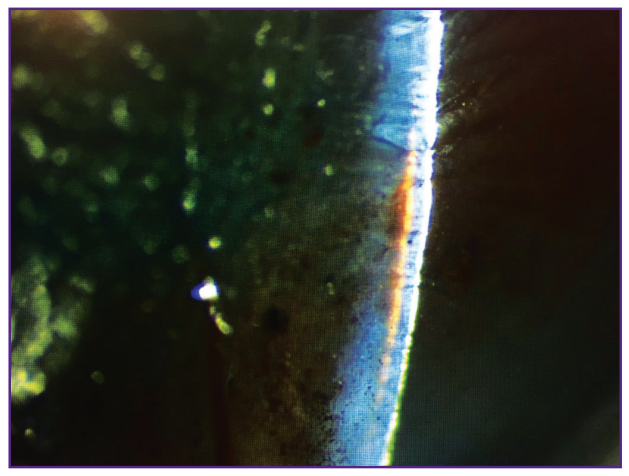

Figure 3. Gel implant compactly positioned in the preformed corneal tunnel of the cadaver eye (Haag-Streit photo slit lamp, Germany)

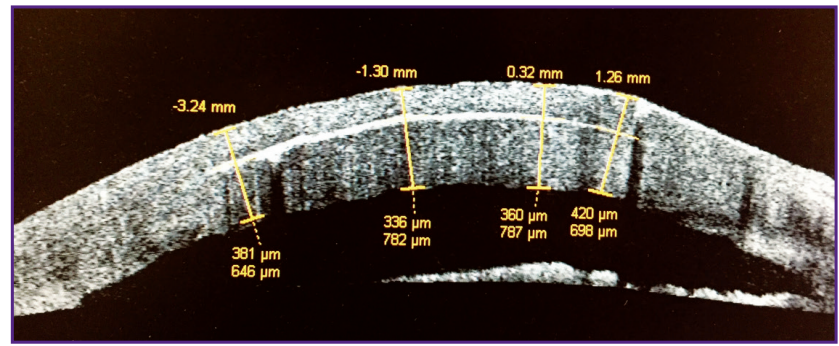

Figure 4. OCT: the gel implant uniformly distributed in the corneal tunnel has a shielding effect 


\section{EXPERIMENTAL INVESTIGATIONS}
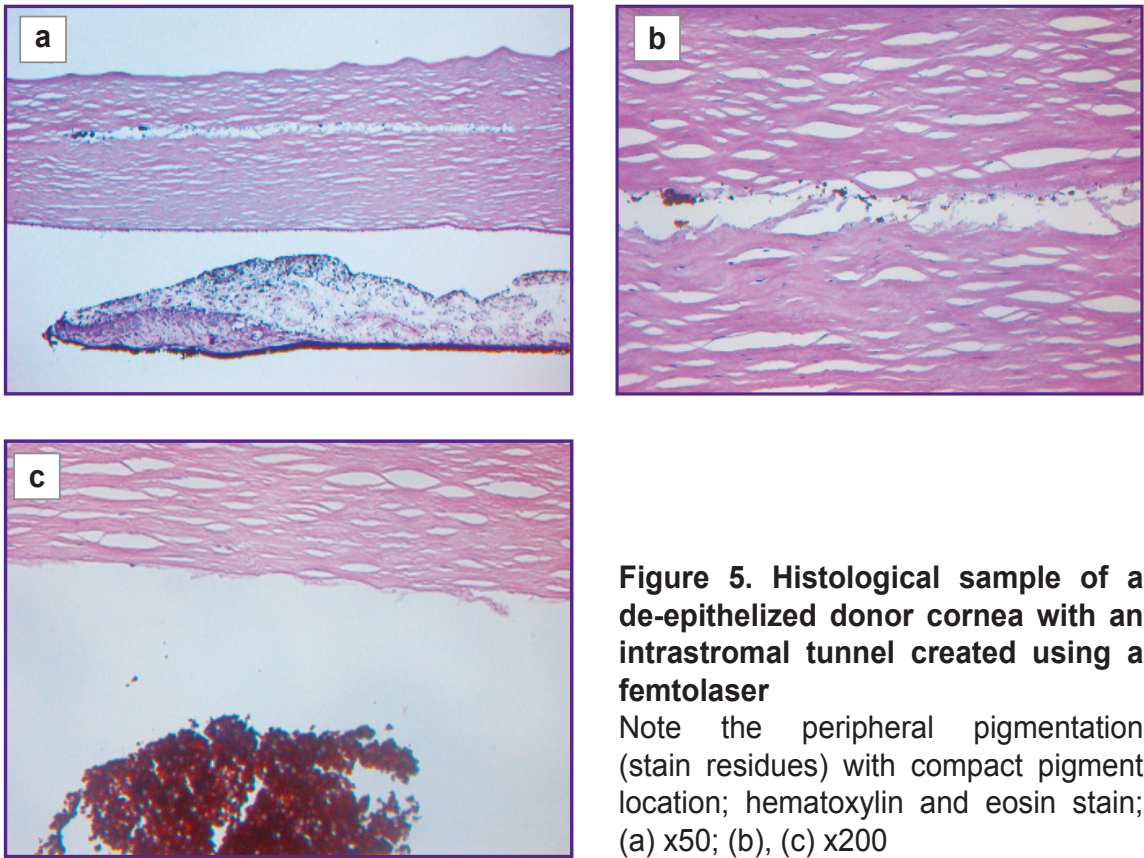

Figure 5. Histological sample of a de-epithelized donor cornea with an intrastromal tunnel created using a femtolaser

Note the peripheral pigmentation (stain residues) with compact pigment location; hematoxylin and eosin stain; (a) x50; (b), (c) x200

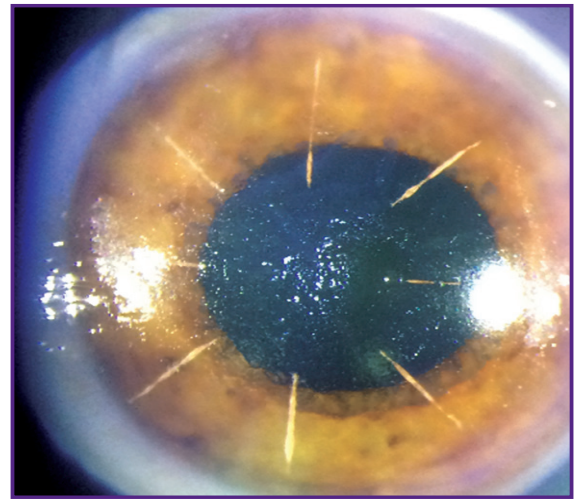

Figure 6. Cadaver eye after radial keratotomy conducted with a femtosecond laser ex vivo
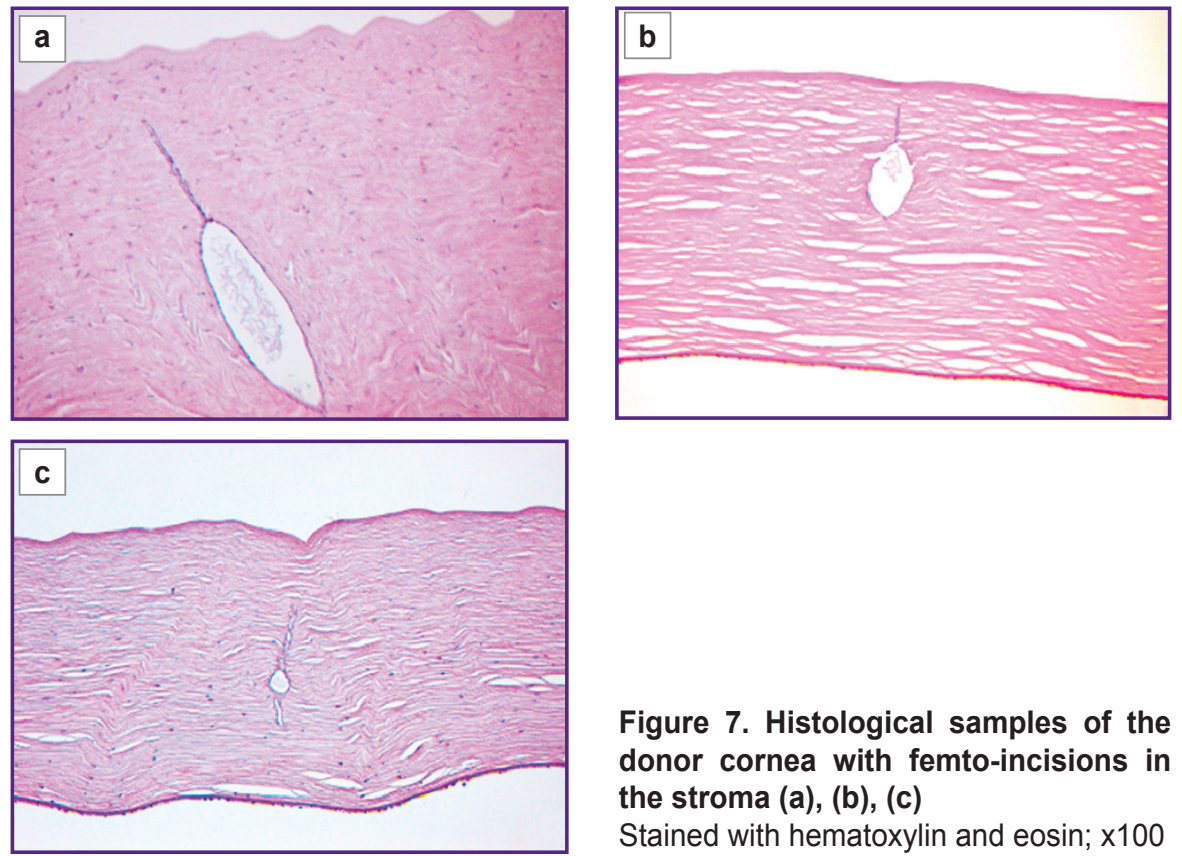

Figure 7. Histological samples of the donor cornea with femto-incisions in the stroma (a), (b), (c)

Stained with hematoxylin and eosin; x100 
Figure 8. Morphology of the incision area after keratotomy made with a diamond blade

Photomicrograph from an Opton photomicroscope (Germany); semi-thin section; stained with methylene blue and magenta [11]

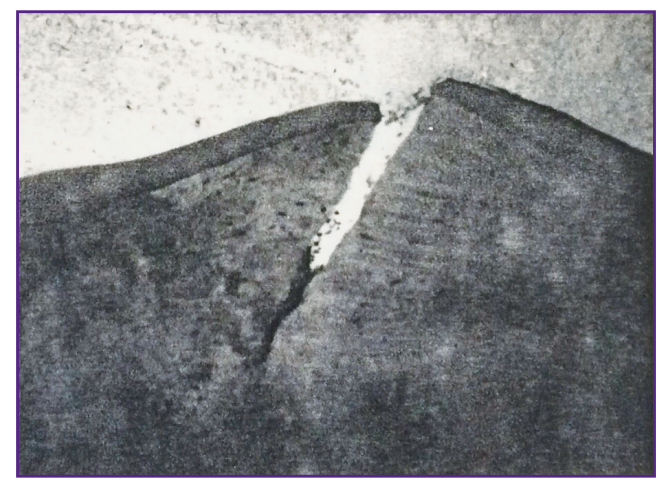

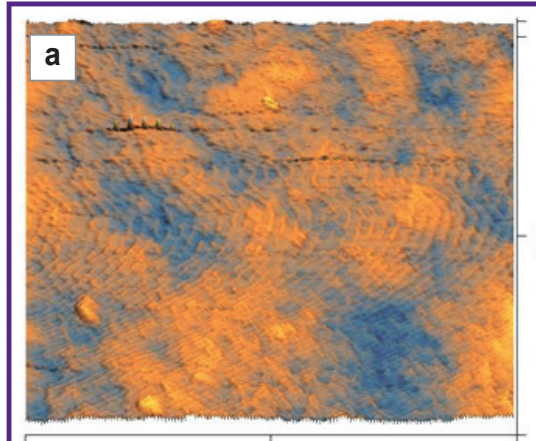

$\mathrm{x}: 5.3 \mu \mathrm{m}$

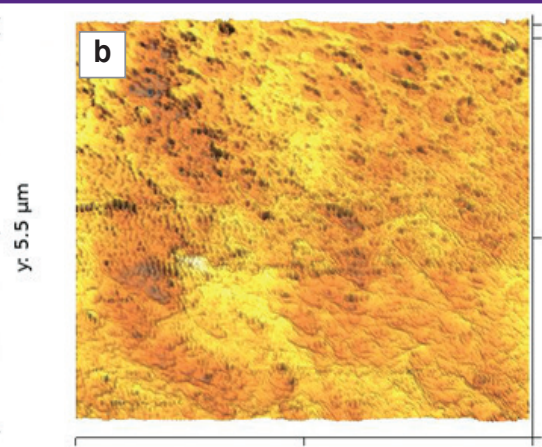

$\mathrm{x}: 6.4 \mu \mathrm{m}$

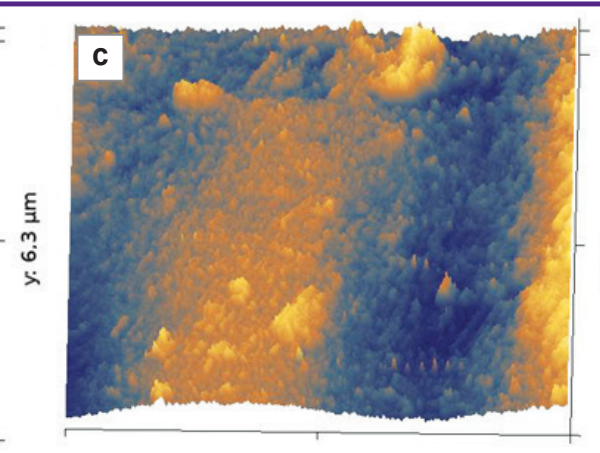

$\mathrm{x}: 5.3 \mu \mathrm{m}$

Figure 9. Atomic force microscopy images of the cornea surface in the control group (a), (b), (c)

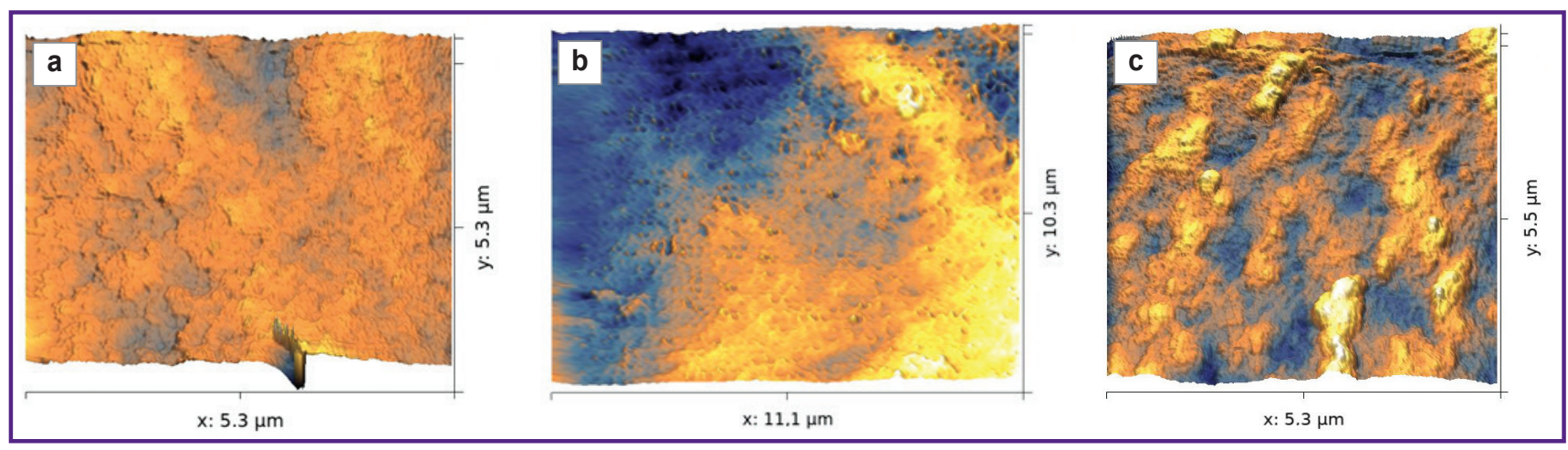

Figure 10. Atomic force microscopy images of the cornea surface in the study group (a), (b), (c)

the front and rear surfaces of the cornea were preserved in all incisions, and through-cutting of the cornea was not observed in any incision (Figure 7). Notably, with the manual radial keratotomy (control group), we found a divergence between the incision edges immediately after the procedure (Figure 8).

The surface quality of the ultrathin graft for posterior lamellar keratoplasty (as determined using atomic force microscopy) showed that the value of the root-meansquare surface roughness in samples prepared with a mechanical microkeratome (control) was $22.3 \pm 18.3 \mu \mathrm{m}$ (Figure 9). The roughness in the study group samples prepared using a femtosecond laser from the endothelial access was $18.72 \pm 12.0 \mu \mathrm{m}$ (Figure 10). Statistical analysis did not reveal significant differences between these two values $(p>0.05)$.

\section{Discussion}

In this study, we obtained the results supporting the technical feasibility of the proposed surgical interventions on the cornea.

The experiments on ISR-assisted keratoplasty confirmed that the pre-formed intralamellar pocket was crucial for stabilizing the ISR implant and preventing its deviation from the pre-set coordinates. Also, the pocket formation made it possible to avoid blanket suturing, which might cause complications (for example, a stitch 
burst due to a close contact between the suture and the ISR surface). Thanks to the original software for the femtosecond laser, we were able to produce the required configuration of corneal sections.

Regarding the intrastromal injection of a colored gel implant we showed that the creation of a corneal tunnel using a femtosecond laser was a precise, predictable and safe process comparable with those reported in the literature [28]. The gel-based structure of the injected substance provides for a stable compact fixation of the implant within the corneal tunnel. According to the results of histological examination, there were no signs of peripheral pigment leakage, which confirmed the stable positioning of the pigment in the corneal tunnel.

The results of this experimental femtolaser keratotomy suggest that incising the Bowman's membrane while preserving the epithelial layer can help avoid the adverse epithelial lining in the incision area that often occurs in the postoperative period and can cause postoperative complications and the refractive effect.

Regarding the ultrathin graft surface quality, the use of the femtosecond laser was found beneficial: its rootmean-square surface roughness was $18.72 \pm 12.0 \mu \mathrm{m}$ (see Figure 2), whereas the same parameter in the samples produced with a mechanical microkeratome was $22.3 \pm 18.3 \mu \mathrm{m}$ (see Figure 1).

\section{Conclusion}

The original software developed for the Femto Visum femtolaser instrument makes it possible to produce corneal sections with the characteristics applicable in many innovative areas of corneal surgery. Simulation of ISR-associated keratoplasty demonstrated that the implanted ring was stably held fixed in its intralamellar pocket along the entire perimeter and therefore no suturing was needed. The quality and accessibility of the gel implantation procedure together with the technical feasibility of its implementation are confirmed by the results of our ex vivo experiments and the histological data. The above advantages will make it possible to create a new, promising, clinically applicable method for treating patients with keratoconus of various stages. According to the degree of roughness, the surface quality of the ultrathin grafts prepared for posterior lamellar keratoplasty using a femtosecond laser was similar to that of the grafts prepared using a mechanical microkeratome. This result indicates the high optical properties of these grafts.

The use of the Femto Visum femtosecond laser as described in the present report is a precise, effective and safe method of surgical interventions on the cornea. Our preliminary results stimulate further research into additional options of using this laser system in clinical practice.

Acknowledgment. The authors are grateful to Mr. A.S. Zavialov, the head of the femtosecond medical lasers group in Optosystems Ltd. for his assistance and personal contribution to the work.

Research funding. The study was supported by personal financial contributions from the authors.

Conflict of interest. The authors declare no conflicts of interest to be reported.

\section{References}

1. Krumeich J.H., Daniel J. Perforating keratoplasty with an intracorneal ring. Cornea 1999; 18(3): 277-281, https://doi. org/10.1097/00003226-199905000-00006.

2. Krumeich J.H., Duncker G. Intrastromal corneal ring in penetrating keratoplasty: evidence-based update 4 years after implantation. J Cataract Refract Surg 2006; 32(6): 993-998, https://doi.org/10.1016/j.jcrs.2006.02.020.

3. Kalinnikov Yu.Yu., Leont'eva G.D., Selifanov Yu.V., Bezzabotnov A.I., Zadorozhnyy S.V. Method of performing keratoplasty (versions). Patent RU 2589633. 2016.

4. Choyce D.P. Semirigid corneal in lays used the managment of albinism, aniridia and ametropia. In: XXIV International Congress of ophthalmology. Acta, San Francisco; 1982; p. 1230-1234.

5. Venger G.E. Vosstanovitel'naya khirurgiya raduzhnoy obolochki pri travmakh glaza $i$ ikh iskhodakh. Avtoref. dis. ... dokt. med. nauk [Iris reconstructive surgery in case of eye injuries and their outcomes. PhD Thesis]. Odessa; 1984.

6. Fedorov S.N., Zuev V.K., Vagrov S.N., et al. Surgical correction of aniridia and iris defects. Oftal'mokhirurgiya 1990; 2 : 20-22.

7. Rodríguez A., Alió J., Amesty M., Bahrawy M. Text and atlas on corneal pigmentation. Jaypee Brothers Medical Publishers (P) Ltd.; 2015, https://doi.org/10.5005/jp/ books/12571.

8. Bowman C.B., Thompson K.P., Stulting R.D. Refractive keratotomy in keratoconus suspects. J Refract Surg 1995; 11(3): 202-206.

9. Grandon S.C., Weber R.A. Radial keratotomy in patients with atypical inferior steepening. J Cataract Refract Surg 1994; 20(6): 670-671, https://doi.org/10.1016/s08863350(13)80171-x.

10. Kopaeva V.G., Leykina S.L., Tekari M. Keratotomiya v korrektsii ametropii pri keratokonuse. V kn.: Mezhdunarododnyy simpozium po refraktsionnoy khirurgii [Keratotomy for the correction of ametropia in keratoconus. In: International symposium on refractive surgery]. Moscow; 1991; p. 54.

11. Ivashina A.I. Khirurgicheskaya korrektsiya blizorukosti metodom peredney radialnoy keratotomii. Dis. ... dokt. med. nauk [Surgical correction of myopia using anterior radial keratotomy. DSc Dissertation]. Moscow; 1989.

12. Lombardi M., Abbondanza M. Asymmetric radial keratotomy for the correction of keratoconus. J Refract Surg 1997; 13(3): 302-307.

13. Abbondanza M., Abdolrahimzadeh B., Guidobaldi M. Combined corneal collagen cross-linking and mini asymmetric radial keratotomy for the treatment of keratoconus. Acta Med Int 2016; 3(1): 63, https://doi.org/10.5530/ami.2016.1.14.

14. Kocak I., Aydin A., Kaya F., Baybora H., Bozkurt S. Efficacy of radial keratotomy in the optical rehabilitation of mild to moderate keratoconus cases. Int Eye Sci 2015; 15(4): 572-576.

15. Fujimoto K., Osawa H., Moriyama T., Miyamoto T., 
Irie T., Nishimura T., Inoue T. Long-term stability of minimally invasive radial keratotomy for mild to moderate keratoconus. Asia Pac J Ophthalmol 2017; 6(5): 407-411, https://doi. org/10.22608/apo.2016204.

16. Utine C.A., Bayraktar S., Kaya V., Kucuksumer Y., Eren H., Perente I., Yilmaz O.F. Radial keratotomy for the optical rehabilitation of mild to moderate keratoconus: more than 5 years experience. Eur J Ophthalmol 2006; 16(3): 376-384.

17. Durand L., Monnot J.P., Burillon C., Assi A. Complications of radial keratotomy: eyes with keratoconus and late wound dehiscence. Refract Corneal Surg 1992; 8(4): 311-314.

18. Panda A., Sharma N., Kumar A. Ruptured globe 10 years after radialkeratotomy. J Refract Surg 1999; 15(1): 64-65.

19. Malyugin B.E., Moroz Z.I., Kovshun E.V., Drozdov I.V. Zadnyaya avtomatizirovannaya posloynaya keratoplastika $s$ ispol'zovaniem ul'tratonkikh transplantatov. $\mathrm{V}$ kn.: IX s"ezd oftal'mologov Rossii: tezisy dokladov [Dorsal automated lamellar keratoplasty using ultrathin grafts. In: IX Congress of Russian ophthalmologists: abstracts of reports]. Moscow; 2010; p. 310.

20. Busin M., Patel A.K., Scorcia V., Ponzin D. Microkeratome-assisted preparation of ultrathin grafts for descemet stripping automated endothelial keratoplasty. Invest Ophthalmol Vis Sci 2012; 53(1): 521-524, https://doi. org/10.1167/iovs.11-7753.

21. Sikder S., Nordgren R.N., Neravetla S.R., Moshirfar M. Ultra-thin donor tissue preparation for endothelial keratoplasty with a double-pass microkeratome. Am J Ophthalmol 2011; 152(2): 202-208.e2, https://doi.org/10.1016/j.ajo.2011.01.051.

22. Cheng Y.Y., Schouten J.S., Tahzib N.G., Wijdh R.J.,
Pels E., van Cleynenbreugel H., Eggink C.A., Rijneveld W.J., Nuijts R.M. Efficacy and safety of femtosecond laser-assisted corneal endothelial keratoplasty: a randomized multicenter clinical trial. Transplantation 2009; 88(11): 1294-1302, https:// doi.org/10.1097/tp.0b013e3181bc419c.

23. Cheng Y.Y., van den Berg T.J., Schouten J.S., Pels E., Wijdh R.J., van Cleynenbreugel H., Eggink C.A., Rijneveld W.J., Nuijts R.M. Quality of vision after femtosecond laser-assisted descemet stripping endothelial keratoplasty and penetrating keratoplasty: a randomized, multicenter clinical trial. Am J Ophthalmol 2011; 152(4): 556-566.e1, https://doi. org/10.1016/j.ajo.2011.03.012.

24. Izmaylova S.B., Malyugin B.E., Novikov S.V., Zimina M.V. Method of carrying out keratoplasty with one-momentary implantation of the intrastomal ring. Patent RU 2646588. 2017.

25. Izmaylova S.B., Novikov S.V., Zimina M.V., Chuprin V.V. Method of carrying out keratoplasty with onemomentary implantation of the intrastomal ring for the prevention of postoperative astigmatism. Application for a patent RU 2018104821. 2018.

26. Izmaylova S.B., Novikov S.V., Sobolev N.P., Yarovoy A.A., Komarova O.Yu. Method of surgical treatment of aniridia with ametropia. Application for a patent RU 2017135291. 2017.

27. Izmaylova S.B., Vartapetov S.K., Konovalova M.M., Burdel K.V. Method for the treatment of keratoconus stage I-III (versions). Application for a patent RU 2018101843. 2018.

28. Alió J.L., Rodriguez A.E., Toffaha B.T., Piñero D.P., Moreno L.J. Femtosecond-assisted keratopigmentation for functional and cosmetic restoration in essential iris atrophy. J Cataract Refract Surg 2011; 37(10): 1744-1747, https://doi. org/10.1016/j.jcrs.2011.08.003. 